\title{
An appetite for learning
}

The hormone leptin is well known for its role in energy homeostasis, but it has also been found to influence synaptic plasticity. Investigating the mechanisms that underlie this function, Moult et al. now show that leptin induces glutamate receptor 1 (GluR1) expression at hippocampal synapses and increases synaptic strength by inhibiting the protein phosphatase and tensin homologue (PTEN).

Synaptic plasticity requires AMPAR (a-amino-3-hydroxy-5methyl-4-isoxazole propionic acid

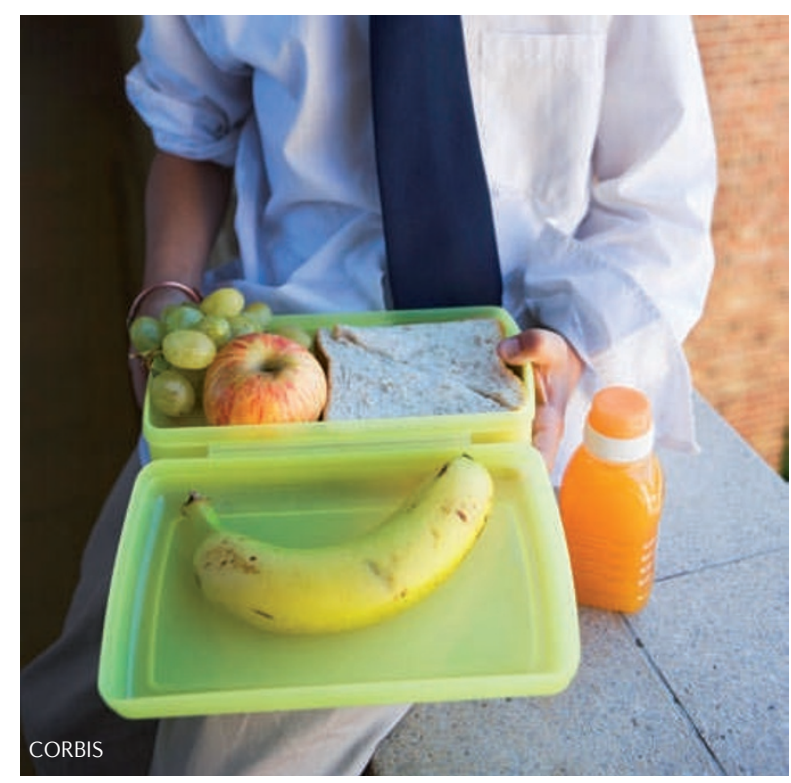

receptor) trafficking, and the authors showed that leptin increased the surface expression of GluR1 AMPAR subunits in hippocampal slices. Leptin did not have this effect in the presence of exocytosis inhibitors or in slices from obese Zucker rats, which are leptin-insensitive owing to a mutation in the leptin receptor gene. These findings indicate that leptin-induced GluR1 surface expression is due to enhanced GluR1 exocytosis and requires leptin receptor activation.

The effect on GluR1 expression had functional consequences: leptin enhanced the amplitude of excitatory postsynaptic currents (EPSCs) in hippocampal slices from wild-type and lean Zucker rats but not in slices from obese Zucker rats.

Immunocytochemical staining revealed that, following leptin application, intracellular phosphatidylinositol-3,4,5-trisphosphate (PtdIns $\left.(3,4,5) \mathrm{P}_{3}\right)$ levels in hippocampal neurons increased before the levels of surface GluR1 did, suggesting that PtdIns $(3,4,5) \mathrm{P}_{3}$ signalling might mediate GluR1 trafficking. As intracellular PtdIns $(3,4,5) \mathrm{P}_{3}$ levels are negatively regulated by PTEN, the authors investigated whether leptin affected the activity of this protein. Indeed, leptin application increased the amount of phosphorylated (that is, inactive) PTEN and led to an increase in PtdIns $(3,4,5) \mathrm{P}_{3}$ levels in hippocampal neurons.

To confirm the possibility that PTEN inactivation underlies the leptin-induced increase in PtdIns $(3,4,5) \mathrm{P}_{3}$ levels, the authors inhibited PTEN in hippocampal neurons by expressing dominantnegative PTEN or by applying the PTEN inhibitor bisperoxovanadium and observed that this increased both surface GluR1 expression and EPSC amplitude. Moreover, adding leptin had no effect on PTENinhibited neurons, indicating that inhibition of PTEN both mimics and occludes the effects of leptin on GluR1 trafficking and synaptic transmission.

These findings shed light on some of the mechanisms by which leptin affects synaptic plasticity. They also suggest that altered leptin signalling or levels, which are found in obesity, diabetes and Alzheimer's disease, might contribute to the cognitive impairment that is associated with these conditions in humans.

Leonie Welberg

ORIGINAL RESEARCH PAPER Moult, P. R. et al. Leptin regulates AMPA receptor trafficking via PTEN inhibition.J. Neurosci. 30, 4088-4101 (2010) 